\title{
Rétrospective 2010: forte implication de la FMH
}

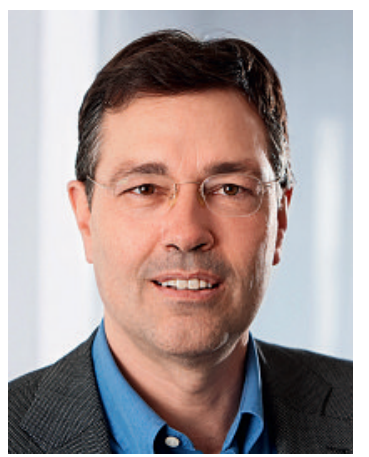

Avez-vous le même sentiment? N'avez-vous pas l'impression que 2010 s'est écoulée encore plus rapidement que les années précédentes? Peut-être est-ce notamment dû au fait que la FMH a été particulièrement active ces derniers 12 mois.

Vous vous en souvenez certainement: la série de mesures d'urgence pour endiguer les coûts de la santé ne nous avait guère laissé de répit en 2009. Et pourtant, des 40 mesures initialement proposées, il n'en est resté qu'un petit nombre en fin d'année dernière, et quelques-unes ont même perdu leur statut d'urgence en 2010. Le mérite d'avoir empêché ces mesures en partie désastreuses revient notamment à la FMH qui a accompli cette année un important travail d'explication, de lobbying et d'intervention.

Le Conseiller fédéral Didier Burkhalter a fixé d'autres priorités que son prédécesseur. Au lieu de mettre l'accent sur la confrontation et des mesures isolées, il appréhende la santé comme un système global et il vise des solutions partenariales. Nous espérons qu'il pourra imposer sa vision. En 2010, l'agitation et des mesures prises au coup par coup ont beaucoup moins perturbé le domaine de la santé.

La révision de la LAMal, et essentiellement les questions du managed care, du libre-choix des patients dans les soins ambulatoires, de l'amélioration de la compensation des risques, etc., a tenu la politique en haleine pratiquement pendant toute l'année - et donc naturellement aussi les organes de la FMH.

$\mathrm{Au} \mathrm{vu} \mathrm{des} \mathrm{intérêts} \mathrm{très} \mathrm{divergents} \mathrm{qui} \mathrm{s'affrontent} \mathrm{en}$ politique, rien d'étonnant à ce que les débats sur ce projet de révision ne parviennent pas à leur terme lors de cette session d'hiver: les questions fondamentales abordées et certains points précis revêtent une signification trop importante, non seulement pour le corps médical mais aussi pour les autres acteurs de la santé. Les solutions choisies auront des répercussions colossales sur tout le système de santé.

Mais en 2010, la politique n'a pas été le seul cheval de bataille de la FMH. Notre organisation professionnelle a aussi mené à bien une série de projets utiles pour le corps médical; en voici quelques-uns:

- Health Professional Card: plus du tiers du corps médical est aujourd'hui titulaire de cette carte. Il s'agit maintenant d'élargir les possibilités d'utilisation de cette nouvelle carte de médecin.

- Monitorage de la qualité: plus de 1500 médecins de famille, psychiatres et orthopédistes exerçant dans le secteur ambulatoire ont déclaré leurs activités en faveur de la qualité dans le cadre du projet «Monitorage de la qualité». La FMH a pour objectif de documenter au niveau national les démarches qualité des médecins du secteur ambulatoire.

- ReMed: après une phase pilote réussie, la FMH a étendu ce réseau de soutien à toute la Suisse. ReMed conseille les médecins en situation de crise en leur proposant une offre personnalisée, axée spécialement sur leurs besoins. En outre, nous ne devons naturellement pas oublier nos activités dans le domaine des tarifs. En effet, la FMH s'est également engagée en 2010 pour des tarifs équitables dans le domaine ambulatoire comme dans le secteur hospitalier. Les points forts, ici, ont été entre autres l'indemnité forfaitaire de dérangement en cas de visite, la remise directe de médicaments, le tarif des analyses, la révision du TARMED mais aussi le dépôt de propositions et la recherche concomitante dans le domaine SwissDRG.

En fin d'année, la question surgit toujours de savoir si nous avons tout fait correctement, si nous avons mis les priorités aux bons endroits? Un regard sur 2010 montre clairement que la FMH sait agir rapidement quand il s'agit de thèmes vitaux pour le corps médical; elle est en mesure de proposer des compromis mais elle sait également défendre ses positions et argumenter avec succès, même contre la résistance d'autres groupes d'intérêt.

Je souhaite à toutes et à tous de belles fêtes de Noël au cours desquelles nous pourrons non seulement méditer sur les choses essentielles de la vie, mais aussi puiser la force et l'énergie pour attaquer la nouvelle année et les nombreux défis que 2011 ne manquera pas de nous apporter.

Daniel Herzog, lic. en droit, MHA Secrétaire général de la FMH 